\title{
Movilidad residencial intraurbana de los españoles y extranjeros en Zaragoza (España): diferentes espacios, distintos procesos
}

\author{
Intra-urban residential mobility of Spaniards and foreigners \\ in Zaragoza (Spain): different spaces, different processes
}

\author{
Severino Escolano-Utrilla ${ }^{*}$ \\ Carlos López-Escolano ${ }^{2}$ (iD \\ Ángel Pueyo Campos ${ }^{3}$ (1)
}

\section{Resumen}

La movilidad residencial es una práctica material y simbólica que forma parte de procesos de producción social del espacio urbano. Las condiciones físicas, sociales y la representación del espacio geográfico en el que se localizan la residencia actual y la futura son factores fundamentales de las decisiones que los individuos y familias toman para cambiar de residencia. Cada grupo social, diferenciado por su nacionalidad, etnia, edad o estatus socioeconómico, valora de forma distinta las ventajas e inconvenientes de cada localización, lo que genera diversos patrones espaciales de movilidad residencial. Aquí se estudian, a varias escalas, las configuraciones funcionales y espaciales de los cambios de residencia del grupo de españoles y extranjeros habidos en Zaragoza entre 2003 y 2017, entendidas como una red relacional de lugares conectados por flujos origen-destino de los cambios de residencia. Se utilizan datos individuales agregados en secciones censales a los que se ha aplicado una metodología que combina procedimientos de análisis espacial y de redes geosociales. Los resultados muestran que las redes de movilidad residencial de ambos grupos tienen diferente organización funcional y se desenvuelven, preferentemente, en distintos espacios. Los efectos de la movilidad residencial se manifiestan en variaciones de población y de la composición sociodemográfica de cada área. La distribución de las distancias de los cambios de residencia de españoles y extranjeros se ajusta a un modelo lognormal, permitiendo predecir con precisión la localización de la residencia futura a partir de la localización de la residencia actual.

Palabras clave: movilidad residencial intraurbana; inmigrantes extranjeros; redes socioespaciales; Zaragoza (España).

\footnotetext{
Abstract

Residential mobility is a material and symbolic practice that is part of social production in urban space. Physical and social conditions and the representation of the geographic space in which the current and future residents are located are fundamental factors in the decisions that individuals and families make when changing residence. Each social group, differentiated by their nationality, ethnicity, age, or socioeconomic status, appraises the advantages and disadvantages of each location in a different way, and

1 Departamento de Geografía y Ordenación del Territorio, Universidad de Zaragoza, España. severino@unizar.es. *Autor para correspondencia

2 Departamento de Geografía y Ordenación del Territorio, Universidad de Zaragoza, España. cle@unizar.es

3 Departamento de Geografía y Ordenación del Territorio, Universidad de Zaragoza, España. apueyo@unizar.es 
this generates different spatial patterns of residential mobility. We study at various scales the functional and spatial configurations of the changes of residence of a group of Spaniards and foreigners that occurred in the city of Zaragoza (Spain) between 2003 and 2017. This is understood as a relational network of places connected by origin-destination flows of changes of residence. Individual data aggregated in census sections have been used with a methodology that combines spatial analysis procedures and geosocial networks. Results show that the residential mobility networks of both groups have different functional organisations in different spaces. The effects of residential mobility are manifested by variations in the volume of the population and the sociodemographic composition of each area. Finally, the distribution of the distances involved in changes of residence of Spaniards and foreigners was well adjusted to a lognormal model, which enables accurately predicting the location of a residence.

Keywords: intra-urban residential mobility; foreign immigrants; socio-spatial networks; Zaragoza (Spain).

\section{Introducción}

Los científicos sociales han estudiado los procesos de la movilidad de la población, a diversas escalas, para conocer las características de quién cambia de residencia (o permanece en la misma), por qué razones lo hace, cómo se producen estos cambios, qué efectos desencadenan y, en bastante menor medida, entre qué lugares se producen (Di Virgilio, 2011; King, 2012), aunque la investigación de la movilidad residencial intraurbana ha recibido menos atención que otras movilidades como, por ejemplo, la internacional (Coulter, Ham \& Findlay, 2015).

La noción «ciclo de vida», que expresa las aspiraciones residenciales de los individuos y familias en sucesivas etapas vitales (Clark \& Dieleman, 1996; Pujadas Rúbies, Prats Boned \& Coll Noguera, 2007), ha articulado buena parte de la investigación sobre la movilidad residencial intraurbana. La creciente complejidad de las formas de la movilidad residencial intraurbana (Coulter et al., 2015; Murayama, Inoue $\&$ Hashimoto, 1997; López-Gay, 2017) _impulsada por los cambios en los estilos de vida y en la composición de los hogares- junto con la diversificación epistemológica habida en las ciencias sociales desde la década de 1990 han enriquecido el marco teórico y los datos empíricos de los estudios de la movilidad residencial (King, 2012).

Las nuevas perspectivas consideran a la movilidad residencial intraurbana como una práctica material y simbólica, de naturaleza relacional, que conecta vidas a través del tiempo y el espacio (Coulter et al., 2015) y que se desarrolla en un contexto en el que interactúan las preferencias y posibilidades individuales con las estructuras socioeconómicas y del espacio urbano. Desde este punto de vista, este tipo de movilidad contribuye notablemente a la transformación de la geografía social, funcional y urbanística de las ciudades (López-Gay, 2014; López-Gay, 2018).

El estudio de la movilidad residencial intraurbana, entendida como un fenómeno social complejo (Torrado, 2020), implica la diversificación de sus enfoques y contenidos así como la consideración de la localización, no tanto como una variable independiente más de un proceso de decisión de cambio de vivienda, sino uno de los primeros condicionantes que actúa en dicho proceso, es decir, las aspiraciones residenciales se buscan primero en las proximidades de la residencia actual e, incluso, se renuncia a algunas por permanecer en las cercanías de esta. La elección de una nueva vivienda en el mismo barrio o área que la vivienda actual supone ventajas como mantener las relaciones sociales, el sentimiento de pertenencia a una comunidad o minimizar el aprendizaje de nuevos espacios urbanos (Sun \& Manson, 2012). Aunque la movilidad residencial intraurbana ha sido poco investigada (Bayona-Carrasco, 2011), estudios empíricos han documentado el tipo e intensidad de la influencia de diferentes factores que impulsan o limitan la movilidad residencial intraurbana. Entre otros, los cambios de residencia se han relacionado con la dinámica y políticas de los mercados de la vivienda (Andrews \& Caldera Sánchez, 2011; Van der Vlist, Czamanski \& Folmer, 2011), el estatus socioeconómico de los sujetos que cambian de residencia, las características del entorno (Bible $\&$ Brown, 1981; Brown \& Moore, 1970; Brummel, 1977), la mejora de la accesibilidad al trabajo o a los servicios y equipamientos públicos (Morris, Manley \& Sabel, 2018), las oportunidades de inversión o cambios en el estilo de vida (Méndez \& Gayo, 2019), con razones emocionales (Raulin, 2014; Villani, 2014) o por vínculos familiares (Tosi, 2017) y otros factores.

También se han publicado investigaciones sobre las características, impacto y pautas de movilidad residencial de grupos específicos de migrantes extranjeros (Thiers-Quintana \& Gil-Alonso, 2019; Zhong \& Beltrán, 2020; Bayona-Carrasco; 2011; Bayona-Carrasco \& López-Gay, 2011) y demostrado la existencia 
de diferencias espaciales en la movilidad residencial de personas blancas y negras (Freeman \& Sunshine, 1976) y entre migrantes y no migrantes (Jia \& Lei, 2019). Sin embargo, han sido poco estudiados los tipos espaciales de movilidad residencial (Torrado, 2020) y las configuraciones territoriales de la movilidad residencial intraurbana. Estos trabajos han permitido identificar patrones espaciales de cambios de residencia agregados a diferentes escalas (White, 1985), caracterizar los impactos de la movilidad residencial, identificar áreas y estructuras socioespaciales (Bayona-Carrasco \& Gil-Alonso, 2012), así como relacionar la localización de origen y destino de los movimientos residenciales con otros factores del proceso de decisión (Brown \& Moore, 1970; Quigley \& Weinberg, 1977). La distancia y dirección de los cambios de residencia han sido las principales variables espaciales estudiadas. Los resultados muestran, de forma consistente, que la mayor parte de los cambios de residencia se producen a corta distancia y que la dirección predominante es hacia la periferia de las ciudades (Potrykowska, 1993; Trépanier, 1978; Bayona i Carrasco \& Pujadas Rúbies, 2010).

En esta línea, la presente investigación aborda el estudio de la estructura y de los patrones espaciales de la movilidad residencial de los españoles y de los extranjeros en la ciudad de Zaragoza (España) entre 2003 y 2017, con enfoque, métodos y unidades espaciales diferentes de los estudios citados.

La utilización de las categorías «españoles» y «extranjeros» y el período estudiado se fundamentan del modo siguiente. Por un lado, varios trabajos citados (Bayona-Carrasco, 2011; Bayona-Carrasco \& López-Gay, 2011; Bayona-Carrasco \& Gil-Alonso, 2012) utilizan estas categorías para demostrar que existen diferencias en las características y efectos de la movilidad residencial entre ambos grupos. Por otro, se puede aceptar que la movilidad residencial del grupo de españoles y la de los extranjeros presentará, con elevada probabilidad, patrones espaciales diferentes puesto que las características demográficas (estructura por edades, composición de los hogares), sociales, económicas, culturales y otros factores que influyen en las decisiones de cambio de residencia, lo hacen de manera distinta en ambos grupos. Este supuesto se puede aplicar también a los inmigrantes de cada continente o nacionalidad, pero lo que aquí se pretende es comprobar si el hecho de ser español o extranjero se refleja de forma consistente en diferencias en el modo de organización y en los espacios de la movilidad residencial de ambos grupos, para avanzar, como línea de trabajo futura, en la investigación de la movilidad residencial de grupos específicos de inmigrantes extranjeros (nacionalidad, edad, etc.). El período seleccionado corresponde a una etapa demográfica y urbanística expansiva, que se inicia con las trasformaciones asociadas a la llegada de la Alta Velocidad Ferroviaria en 2002 (AVE) (Alonso Logroño \& Bellet Sanfeliu, 2009) y continúa con desarrollos asociados a la Exposición Internacional (2008) (Gómez Bahíllo \& Sanaú Villarroya, 2009) y otros grandes proyectos. La duración de quince años se considera tiempo suficiente para identificar tendencias espaciales significativas de la movilidad residencial.

La organización espacial de la movilidad residencial se conceptúa aquí como un conjunto relacional de lugares conectados por las trayectorias de origen y destino de los cambios de residencia. La intensidad de los flujos de cambio de residencia entre estos lugares define los territorios de la movilidad residencial. La emergencia de la organización espacial de la movilidad residencial se fundamenta en dos supuestos: i) el de autocorrelación espacial, es decir, los cambios residenciales entre espacios próximos tienen alta probabilidad de presentar propiedades similares, y ii) el de homophily, que permite aceptar que los cambios de residencia de personas con estándares sociales parecidos (estatus económico, nivel educativo, modelo familiar, etc.) es muy probable que muestren patrones espaciales semejantes, independientemente de su residencia actual.

El método aplicado combina el análisis espacial y el análisis de redes geosociales para identificar la organización espacial de la movilidad. Las técnicas del análisis de redes geosociales solo se han aplicado de forma excepcional a datos de movilidad residencial intraurbana (Sun \& Manson, 2012) a pesar de su gran potencial para explicar procesos socioespaciales (Andris, Liu \& Ferreira, 2018; Ducruet \& Beauguitte, 2014; Liu, Hollister \& Andris, 2018; Luo \& MacEachren, 2014).

Con este contexto, cabe preguntarse por las similitudes y diferencias de los patrones espaciales de la movilidad residencial de los españoles y extranjeros en el marco de los procesos simultáneos de expansión y fragmentación física, social y funcional detectados en la periferia y centro urbano (Escolano-Utrilla, López-Escolano \& Pueyo-Campos, 2018). Este objetivo comprende dos ámbitos de análisis conectados:

i) El correspondiente a la organización espacial y funcional de la movilidad residencial de españoles y extranjeros a varias escalas en el período 2003-2017. Para este fin se analizará: a) el grado de concentración espacial de los orígenes y destinos de los cambios de residencia (centralidad-hub); 
b) forma de los flujos mutuos entre secciones (ciclos); c) los tipos espaciales de movilidad definidos por áreas socioespaciales de origen y destino de los flujos migratorios y d) el modelo de distribución de las distancias de los cambios de residencia.

ii) El relativo a la configuración espacial de los territorios de la movilidad residencial de ambos grupos, que resultan de las conexiones entre las secciones de origen y destino del cambio de residencia (módulos, agrupamientos o clústeres).

Este trabajo pretende contribuir a mejorar el conocimiento de la movilidad residencial entendida como un fenómeno socioespacial relacional que puede modelarse mediante redes geosociales (socioespaciales) que son expresión y resultado, al mismo tiempo, de procesos sociales y espaciales específicos. Por otra parte, la delimitación de áreas en función de la movilidad residencial es una aportación útil en sí misma, pues supone la aplicación de criterios dinámicos para explorar la estructura socioespacial de las ciudades frente al uso tradicional de atributos estáticos (Sun \& Manson, 2012). Esta información también es relevante para la formulación de políticas públicas en las principales áreas de la planificación y gobernanza urbanas.

El resto del artículo está organizado del modo siguiente. En la segunda sección se describe la metodología, incluyendo las fuentes de datos y métodos empleados junto a las características del área de estudio — la ciudad de Zaragoza-. A continuación, se presentan los resultados obtenidos. Después, se discuten y explican los resultados. Finalmente, se exponen las conclusiones y se proponen líneas de investigación futuras.

\section{Metodología}

\subsection{Datos y métodos}

Los datos utilizados en esta investigación expresan el número de personas empadronadas en 2017 en Zaragoza que han cambiado de sección censal de residencia dentro del municipio de Zaragoza respecto de la sección en la que residían en 2003. Se han obtenido a partir de la agregación de los cambios de residencia individuales registrados en el Padrón Municipal de Habitantes. De cada cambio de residencia se ha registrado la nacionalidad y el sexo. Las unidades de análisis son, pues, los flujos de personas españolas y extranjeras y las secciones censales.

En la mayor parte de los estudios de movilidad residencial se han analizado los patrones territoriales de la movilidad residencial a partir de balances y tasas calculados para unidades espaciales administrativas (generalmente municipios) o macroáreas como las coronas metropolitanas u otras unidades como los barrios. En este estudio se han utilizado unidades espaciales de bastante resolución, secciones censales, y se han identificado las configuraciones espaciales de la movilidad residencial, a varias escalas, definidas por la intensidad de los intercambios residenciales entre las secciones.

La considerable resolución espacial de las secciones censales hace que sean unidades espaciales muy apropiadas para el estudio, a mesoescala, de la movilidad residencial en las ciudades españolas. Esta fuente es bastante fidedigna, ya que el empadronamiento es un requisito para recibir diversas prestaciones sociales, sanitarias y educativas. La localización del origen y destino de los cambios de residencia registrados es muy exacta y precisa. Se ha variado la delimitación de varias secciones censales para homogeneizar los datos de 2003 y 2017.

A esta información se le ha aplicado los procedimientos necesarios para alcanzar los objetivos, en una secuencia lógica que comprende dos fases: a) elaboración de las redes socioespaciales de la movilidad de los españoles y extranjeros y b) análisis, a varias escalas, de la organización funcional y espacial de la movilidad de ambos grupos.

Con los flujos de la movilidad residencial por secciones censales se han formado dos matrices origen-destino que recogen los cambios de residencia entre cada una de las 487 secciones. A partir de cada matriz se ha elaborado una red geoespacial para cada grupo del estudio en la que los nodos representan a las secciones y su localización (los centroides de las secciones) y las aristas (conexiones) simbolizan la dirección, origen-destino y la magnitud de los cambios residenciales entre los nodos. Los nodos contienen información espacial y temática. Esta estructura de datos constituye el modelo de la movilidad residencial de los españoles y extranjeros.

La organización funcional y espacial de la movilidad residencial se ha caracterizado por varios indicadores agrupados en dos niveles: en primer lugar, se analiza la función de cada sección (nodo) en 
el conjunto de la red (valor de centralidad-hub, formación de ciclos o circuitos, tipologías espaciales y distancias de los cambios de residencia); después, se delimita la configuración funcional y territorial de los flujos de la movilidad residencial mediante la identificación de los módulos de la red de flujos de movilidad residencial (clústeres, agrupamientos).

Para describir la estructura de las redes se han seleccionado un indicador de centralidad y otro de conexión entre nodos por su utilidad para los objetivos del trabajo. El primero es la centralidad-hub, que se estima a partir de la centralidad eigenvector. Un nodo (centroide de sección) con valor de centralidad-hub elevado indica que sus conexiones hacia afuera (personas que dejan de residir en esa sección) se dirigen a nodos que tiene valores altos de conexiones hacia dentro (personas que fijan su residencia en esa comuna), es decir, que las secciones con elevados valores de centralidad-hub actúan como distribuidoras de la movilidad residencial. La centralidad-hub se ha calculado con el programa ORA Net-Scenes (Carley, 2017) del modo siguiente:

Centralidad eigenvector: vector combinado de todos los valores escalados Vk de una red. Sean: A una red unimodal de $\mathrm{N}$ nodos; $\mathrm{K}$ el número de componentes de la red $\mathrm{A} ; \mathrm{Nk}$ nodos del componente $k t h ; \mathrm{Vk}$ eigenvector dominante de la subred inducida por los nodos $\mathrm{Nk}$. Se escalan los valores Vk multiplicándolos por Nk/N (Bonacich, 1972). Centralidad-hub: centralidad eigenvector de la red A*A; (Min =0; Max. =1) (Kleinberg, 1998).

Los ciclos son conjuntos de nodos de tal forma que para cada nodo existe una ruta de retorno a ese nodo (por ejemplo, del nodo A al B, del B al C, del C al A). Los ciclos se corresponden con grupos de secciones con intercambios mutuos de residentes.

A escala metropolitana, Torrado (2020) distingue tres formas principales de movilidad residencial, cada una con implicaciones propias en los procesos de reconfiguración metropolitana: los movimientos de suburbanización, que se producen entre el centro y la franja externa de crecimiento metropolitano; los de centralización, desde la corona exterior hacia la ciudad central; y la movilidad entre los municipios de la corona. A estas tres formas de movilidad residencial, que nos parece adecuado mantener en el caso de Zaragoza, se han añadido otras, que surgen de los cambios de residencia entre los ámbitos socioespaciales delimitados. En consecuencia, se han identificado los siguientes tipos de movilidad: a) movimientos de centralización: los que se dirigen al centro desde las demás áreas; b) movimientos internos del área central (algunos de recentralización): tienen su origen y destino en el centro; c) movimientos internos en los barrios (c1, de la margen derecha; $c 2$, de la margen izquierda); d) movimientos entre barrios de la margen derecha e izquierda; e) movimientos de suburbanización: son los que tienen como destino las periferias y su origen en las demás áreas; f) movimientos internos en las áreas suburbanas: son los que se producen dentro y entre las periferias.

Las distancias de los cambios de residencia se han estimado a partir de la distancia euclidiana que separa los centroides (nodos) de cada sección. La distribución nula de las distancias (H0) se ha calculado también con distancias euclidianas en el supuesto de que la población de cada sección se desplaza a todas las demás secciones. El ajuste de las distribuciones empíricas a una distribución teórica se ha llevado a cabo con el programa XLSTATS (como complemento de Excel) por el procedimiento de máxima verosimilitud. El análisis espacial y la cartografía se han realizado con el programa ArcGIS 10.5 (ESRI).

Finalmente, entre los diversos métodos de detección de la composición en módulos (agrupamientos, clústeres) en redes complejas (Malliaros \& Vazirgiannis, 2013), se ha optado por el algoritmo de agrupamiento jerárquico de nodos basado en la optimización de una función de ajuste local (OSLOM), que expresa la significación estadística de los agrupamientos respecto de fluctuaciones aleatorias (Lancichinetti, Radicchi, Ramasco \& Fortunato, 2011). Este método es muy apropiado a las características de las redes aquí estudiadas, ya que puede aplicarse a redes con dirección (directed networks) y con conexiones ponderadas por pesos (los flujos de movilidad), detecta niveles jerárquicos, la pertenencia de nodos a varios agrupamientos, identifica clústeres de diversas configuraciones y deja sin clasificar aquellos nodos que reparten sus flujos de forma equitativa entre el resto de los clústeres. Este algoritmo se ha utilizado para delimitar clústeres funcionales y espaciales definidos por los flujos de trabajo diario en el Reino Unido (Ratti et al., 2010). Los cálculos se han llevado a cabo con el programa Cytoscape 3.7 (Shannon et al., 2003).

El índice E-I es un indicador normalizado de la cohesión de cada módulo que expresa la proporción de intercambios de residencia entre las secciones del mismo módulo (conexiones internas: I) y los intercambios de residencia entre secciones que forman parte de otros módulos (conexiones externas: E). Su ecuación se puede generalizar para utilizar los pesos (migrantes: w) de las conexiones: 


$$
\mathrm{E}-\mathrm{I}=\left(\mathrm{E}_{\mathrm{w}}-\mathrm{I}_{\mathrm{w}}\right) /\left(\mathrm{E}_{\mathrm{w}}+\mathrm{I}_{\mathrm{w}}\right)
$$

Su valor varía entre - 1 (todas las conexiones son internas, es un grupo aislado) hasta 1 (todas las aristas son externas); mientras que el valor 0 indica el mismo número de aristas internas y externas (o idénticos flujos de movilidad residencial entre las secciones del mismo módulo y entre estas y las de los restantes módulos).

La aplicación de esta metodología ha permitido delimitar agrupamientos que desvelan diferentes niveles de organización funcional y espacial de los cambios residenciales e, indirectamente, la actuación de factores subyacentes que generan las interacciones, dentro de cada clúster y con los restantes.

Tanto los datos como los métodos utilizados presentan ciertas limitaciones. En primer lugar, es necesario advertir que los datos subestiman la movilidad residencial entre los dos años de referencia, pues algunas personas han podido mudarse de domicilio varias veces; tampoco se ha contabilizado los cambios de residencia dentro de la misma sección. Estimamos, no obstante, que la muestra con la que trabajamos es suficiente para identificar y delimitar los patrones de la movilidad residencial en el período estudiado. Por otro lado, existen múltiples métodos para delimitar y jerarquizar las áreas de movilidad (clusterización). Por esta razón, se han aplicado varios procedimientos de agrupamiento (Moduland; Szalay-Beko et al., 2012) que han producido resultados ligeramente diferentes a los presentados aquí, pero en todos los casos muestran la existencia de patrones y jerarquías espacialmente coherentes.

\subsection{Caracterización del área de estudio}

La ciudad de Zaragoza ha experimentado profundas transformaciones de su geografía social, espacial y funcional durante los años transcurridos del siglo XXI. La población ha registrado un crecimiento del 9,5\% en el periodo de estudio 2003-2017, y roza los 700.000 habitantes en 2017. El crecimiento del contingente de extranjeros ha sido mayor, pues su número se ha duplicado durante el quindenio de estudio (43.040 habitantes en 2003 y 93.129 en 2017), registrando un crecimiento del 116,4\%, mientras que en el mismo periodo la población española únicamente aumenta en 6.892 personas. Una evolución similar se observa en el conjunto de España para el mismo período, cuando la población total crece un 8,3\% y la población extranjera lo hace en un $41,7 \%{ }^{4}$, aunque es una cifra menos elevada que la registrada en Zaragoza. Sin embargo, este incremento demográfico ha sido desigual en el tiempo y en el espacio de acuerdo con los ciclos económicos y migratorios: i) un periodo inicial (2003-2008) de expansión demográfica y urbana orientada hacia la dispersión en la periferia (Callizo-Soneiro, 2017; De Miguel González, 2015) y ii) otro ciclo posterior (2008-2017) de reorganización interior y escaso desarrollo urbano y estabilidad de la población como en otras ciudades españolas (Martori, Madariaga \& Oller, 2016).

En conjunto, Zaragoza presenta algunas características de ciudad intermedia a escala espacial y demográfica: escala humana que permite el conocimiento y experiencia personal de gran parte del espacio urbano; tamaño de población intermedio en relación a las ciudades del país; composición de la población semejante a la media nacional; rango relevante en el entramado político-administrativo del país; procesos metropolitanos a escala moderada; procesos de suburbanización, dispersión y fragmentación espacial (Escolano-Utrilla et al., 2018); creación de centralidades en la periferia; buena calidad media de vida; o fácil acceso al centro urbano caminando o en transporte público para gran parte de la población (ArranzLópez, Soria-Lara, López-Escolano \& Pueyo Campos, 2017).

En Zaragoza, las migraciones centro-periferia han sido el vector principal de la redistribución de la población en los últimos años (42.318 nuevas viviendas construidas entre 2003 y 2017, IAEST) debido a la disponibilidad de suelo para urbanizar en torno a la ciudad compacta, lo que ha limitado la dispersión de población en otros municipios del área metropolitana respecto a lo sucedido en otras ciudades españolas (Jiménez, Hidalgo, Campesino \& Alvarado, 2018). Hasta estos espacios se ha trasladado mayoritariamente población joven en edad de emancipación procedente de los barrios centrales de la ciudad con estatus socioeconómico medio-alto, mientras que una parte muy significativa de la población de origen extranjero se ha asentado en barrios centrales donde el envejecimiento ha dejado libres numerosas viviendas económicamente más accesibles, y más deterioradas, frente a las nuevas ubicadas en la periferia (Ebrópolis, 2019).

4 Instituto Nacional de Estadística, Estadística del Padrón Continuo. 
La ciudad actual se puede caracterizar por cinco grandes tipos socioespaciales, que se utilizan exclusivamente con fines de descripción e interpretación; son las siguientes: i) Centro urbano: comprende el espacio situado entre el río Ebro y el primer cinturón (muralla medieval) incluyendo el Casco Histórico, así como los ensanches más nobles; aquí se localiza el principal entorno comercial y de negocios de la ciudad; ii) Barrios populares de la margen derecha del río Ebro: se desarrollan entre el centro histórico hasta el cinturón Z-30, también se incluyen los barrios tradicionales del «extrarradio», Oliver y Valdefierro; iii) Barrios populares de la margen izquierda del río Ebro: conforman un espacio cercano al centro urbano entre el río Ebro y el cinturón Z-30; iv) Periferia próxima: corresponde al espacio urbano desarrollado entre los cinturones Z-30 y Z-40, caracterizado por su notable fragmentación espacial, la presencia de condominios de clase alta y la mezcla de usos del suelo y de densidades; y v) Periferia lejana: designa al espacio urbano construido entre el cinturón Z-40 y los límites del municipio. Sus características son similares a las del tipo anterior y además añade algunos núcleos rurales tradicionales.

Finalmente, la expresión "espacio urbano tradicional" se emplea aquí para designar a la ciudad existente hasta mediados de la década de 1980, es decir, aproximadamente el espacio comprendido dentro del cinturón Z-30 y los barrios Oliver y Valdefierro.

Figura. 1. Estructura urbana de la ciudad de Zaragoza

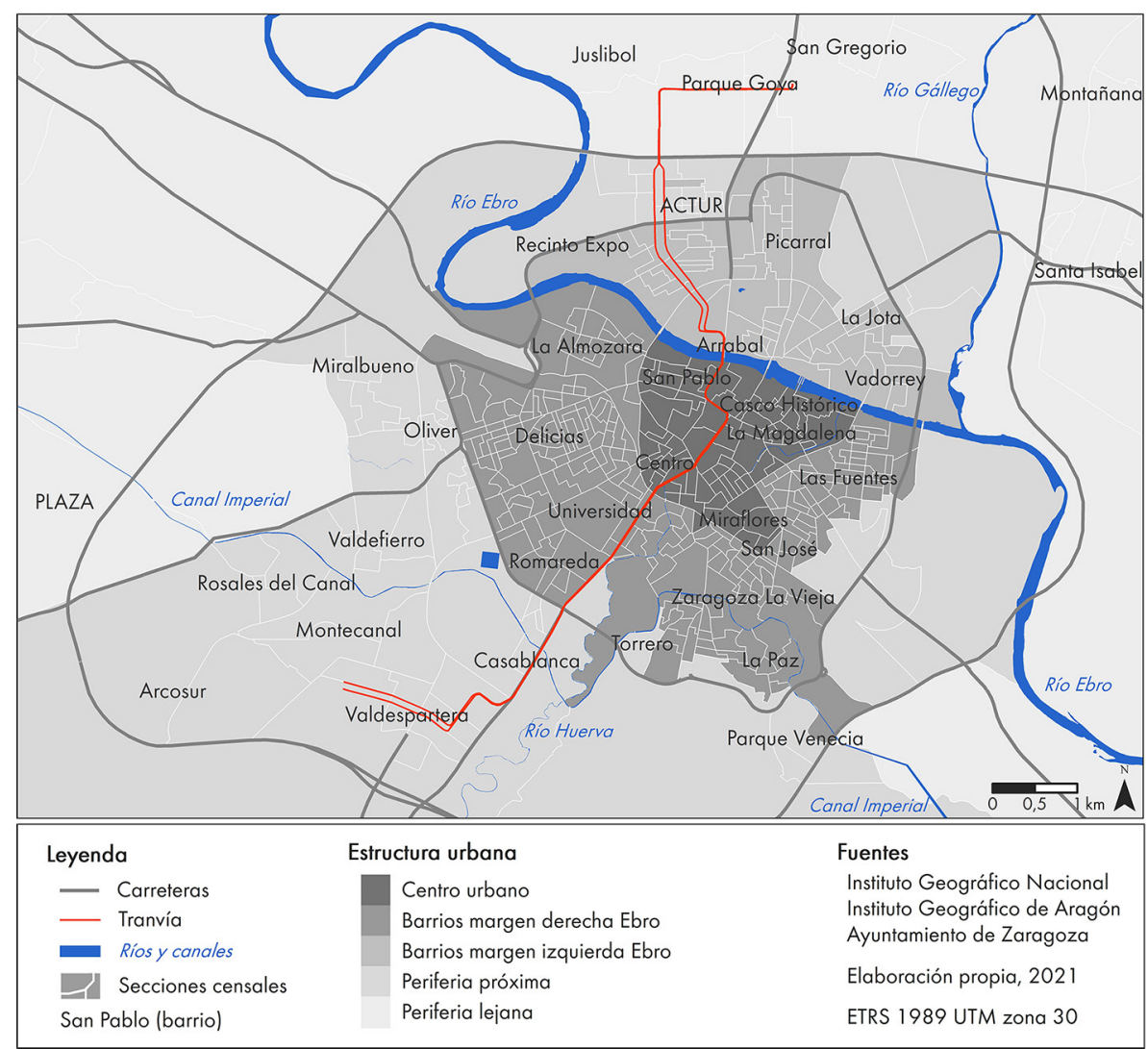

Fuente: Padrones Municipales de Habitantes del Ayuntamiento de Zaragoza. Elaboración propia

\section{Resultados}

En 2017, más de 150.000 personas habían cambiado su domicilio a otra sección censal del municipio de Zaragoza respecto de la que habitaban en 2003, la mayor parte de las cuales (87\%) tenían nacionalidad española y el resto extranjera, lo que supone alrededor del 23\% de la población total de 2003. Si bien, como se recoge en la tabla 1 , los valores relativos de esta variación residencial intraurbana son similares para ambos grupos de estudio, se observa que la proporción de personas que permanecen en la misma sección es considerablemente más elevada en el caso de los españoles $(55,3 \%)$ que en el de los extranjeros $(7,1 \%)$. Ambos hechos indican que buena parte de la movilidad residencial de los extranjeros se ha dirigido a otros lugares fuera del municipio de Zaragoza. 
Tabla 1. Principales indicadores de la movilidad residencial intraurbana de los españoles y de los extranjeros en Zaragoza, 2003-2017

\begin{tabular}{|l|r|r|r|}
\hline & \multicolumn{1}{|c|}{ Españoles } & \multicolumn{1}{c|}{ Extranjeros } & \multicolumn{1}{c|}{ Total } \\
\hline Población total, 2003 & 598.541 & 43.040 & 641.581 \\
\hline Población total, 2017 & 605.620 & 94.084 & 699.704 \\
\hline Cambio de sección de residencia entre 2003 y 2017 (personas) & 130.888 & 19.587 & 150.475 \\
\hline Permanecen en la misma sección entre 2003 y 2017 (personas) & 325.082 & 6.214 & 331.229 \\
\hline Número de secciones & & & 487 \\
\hline $\begin{array}{l}\text { Número de secciones con balance migratorio neto (inmigrantes-emigrantes) } \\
\text { positivo }\end{array}$ & 118 & 258 & \\
\hline $\begin{array}{l}\text { Número de secciones con balance migratorio neto (inmigrantes-emigrantes) } \\
\text { negativo }\end{array}$ & 371 & 220 & \\
\hline Número de conexiones entre secciones & 59.313 & 11.757 & \\
\hline Personas/conexión promedio; mínimo; máximo & 2,$2 ; 1 ; 279$ & 1,$66 ; 1 ; 30$ & \\
\hline
\end{tabular}

Fuente: Padrones Municipales de Habitantes del Ayuntamiento de Zaragoza. Elaboración propia

La intensidad relativa de los cambios de residencia presenta patrones idénticos a los de los flujos absolutos: en el caso de los españoles los valores más elevados corresponden a secciones de la periferia, las zonas de expansión de los barrios de la margen izquierda del Ebro y algunas del área central (los espacios más renovados del Casco Histórico) y en el de los extranjeros a determinadas secciones del Casco Histórico (San Pablo) y de los centros de los barrios (especialmente de Delicias, Las Fuentes y San José) (Figura 2).

Figura. 2. Intensidad relativa de los cambios de residencia por secciones censales de españoles y extranjeros en Zaragoza, 2013-2017

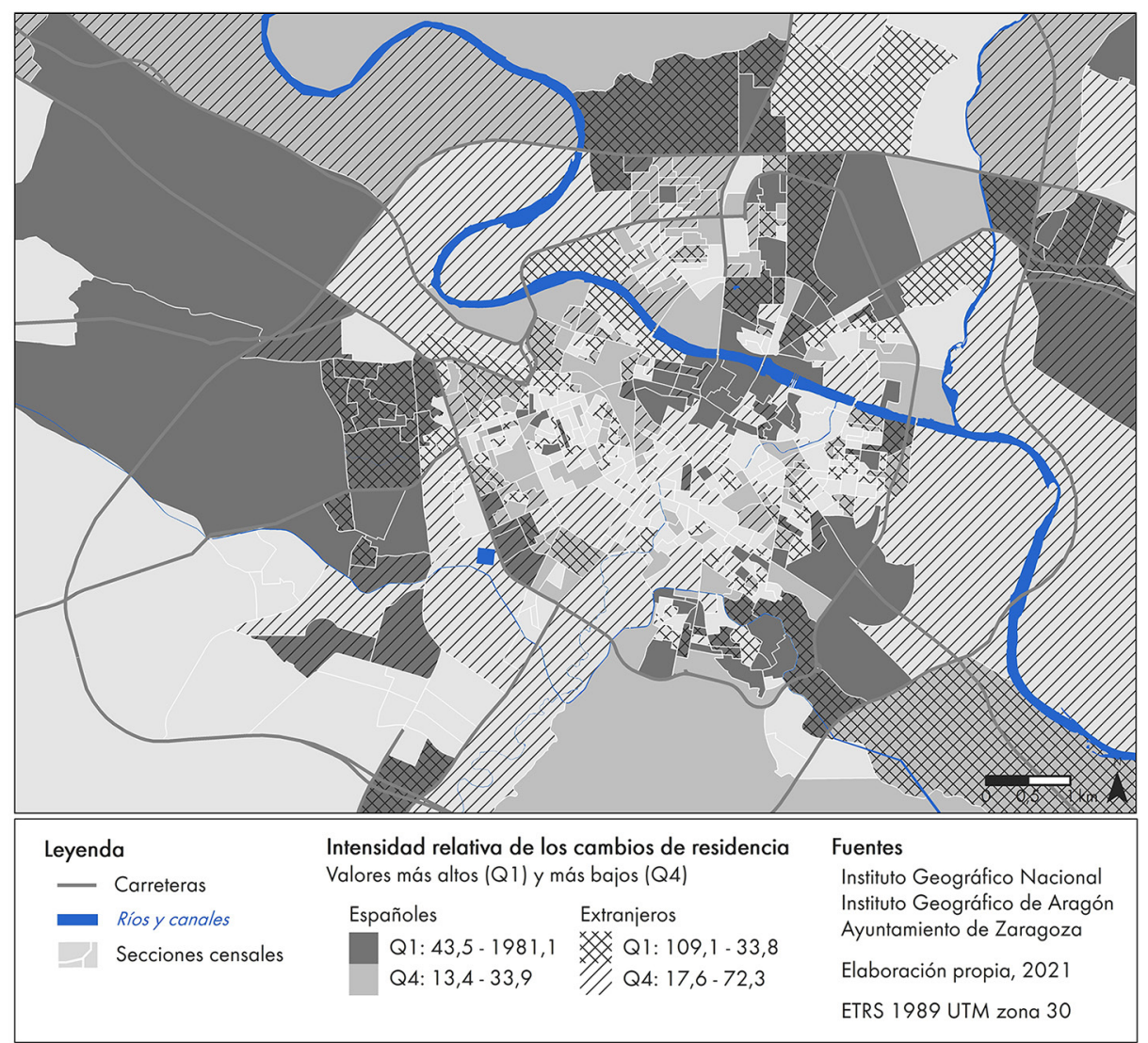

Fuente: Padrones Municipales de Habitantes del Ayuntamiento de Zaragoza. Elaboración propia 
Las redes de movilidad de ambos grupos son diferentes en tamaño, definido por el número de nodos y conexiones, así como en el valor medio de los flujos de movilidad, debido a que el número total de migrantes residenciales del grupo de españoles es muy superior al del grupo de extranjeros.

El mapa de la Figura 3 muestra que el grueso de los flujos de la movilidad residencial de ambos grupos se despliega en espacios diferentes: los barrios al sur de la ciudad para los españoles y los espacios del centro urbano para los extranjeros, con concentraciones significativas para ambos grupos en determinados sectores de la periferia de la ciudad. Los balances netos positivos de la movilidad presentan una distribución claramente periférica.

Figura. 3. Flujos residenciales de españoles y extranjeros en Zaragoza, 2013-2017 (mayores de 10 migrantes españoles y mayores de 5 migrantes extranjeros)

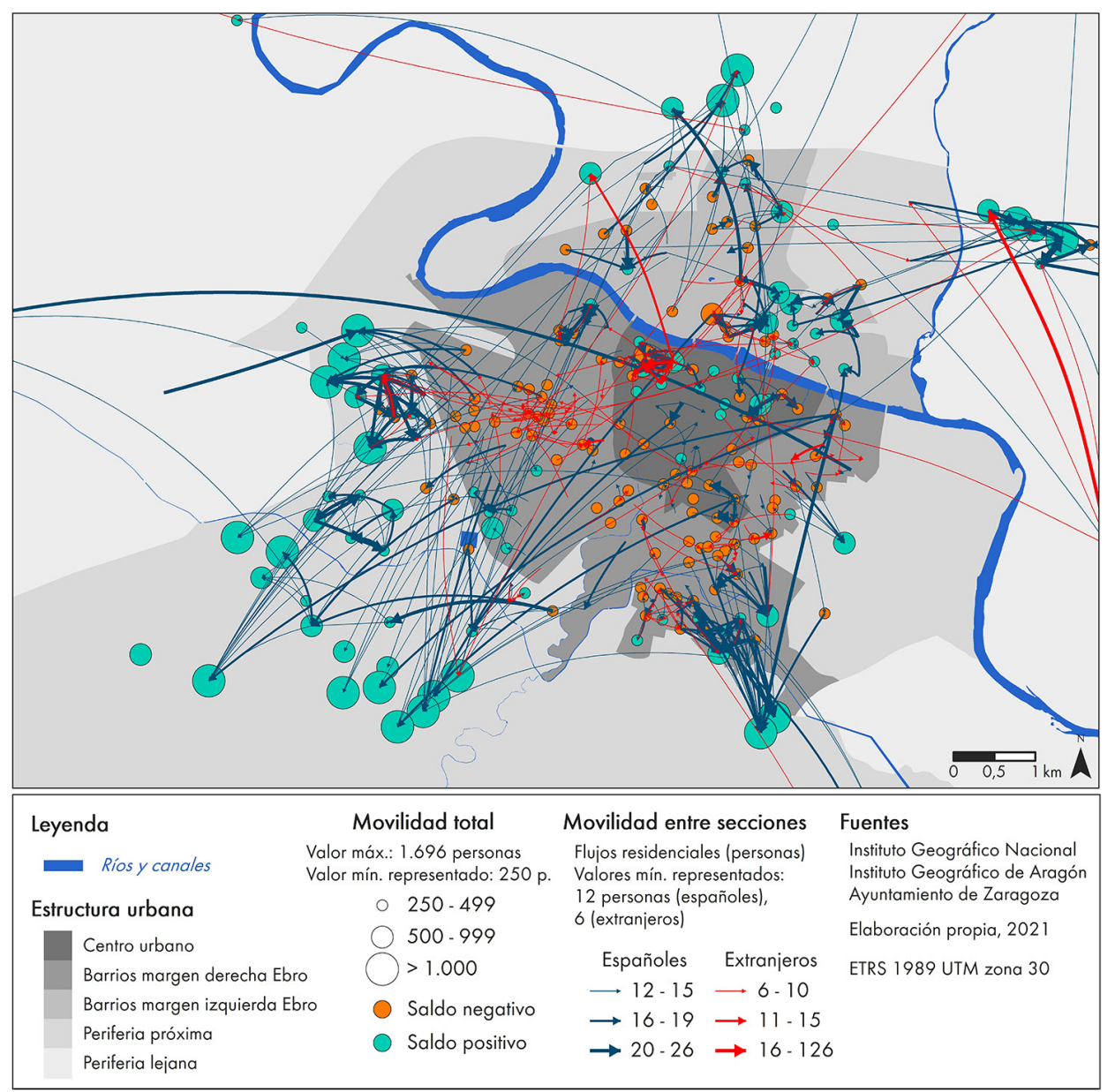

Fuente: Padrones Municipales de Habitantes del Ayuntamiento de Zaragoza. Elaboración propia

En el mapa se aprecia que la distribución espacial de la movilidad residencial no es ni homogénea ni aleatoria, pues presenta sectores con densidades diferentes de cambios de residencia que generan grupos y subgrupos jerarquizados de secciones censales que configuran territorios de movilidad residencial a varias escalas.

Para describir la organización espacial de la movilidad residencial se ha seguido un procedimiento de abajo-arriba: en primer lugar, se estima la funcionalidad de cada sección en el conjunto de la red mediante sus valores de centralidad; después se examinan las conexiones mutuas entre secciones mediante la cuantificación de un patrón de interconexión (motivo); a continuación, se elaboran las tipologías espaciales de la movilidad residencial y se modela la distribución de las distancias que separan la residencia habitual de la nueva. Finalmente, a partir de la configuración de la red de flujos origen-destino y su intensidad se delimitan los territorios de la movilidad residencial a partir de los agrupamientos (clústeres) de los nodos (secciones) de las redes de movilidad. 


\subsection{Diferencias y similitudes de las redes de movilidad residencial de los españoles y extranjeros}

La centralidad-hub mide la capacidad de cada sección en el conjunto de la red de flujos como centro de distribución de la movilidad: de las secciones con elevada centralidad-hub salen residentes que se dirigen a otras secciones a las que también llegan bastantes nuevos residentes. La estructura de la distribución estadística de la centralidad-hub es similar en ambos grupos de estudio, aunque los valores más elevados se reparten de forma más equitativa en el caso de los extranjeros, pues son 23 las secciones que están por encima de 1,5 desviación típica (suman el 21,3\% de la centralidad-hub total) frente a las 6 secciones de los españoles (suman el 4,6\% de la centralidad-hub total). Esta distribución traduce el hecho de que los destinos de la movilidad residencial de los españoles están más concentrados que los de los extranjeros. En cambio, la distribución espacial de estos valores difiere notablemente entre el grupo de españoles y el de extranjeros, como se representa en el mapa de la Figura 2: en el caso de los españoles las secciones distribuidoras se localizan en la periferia mientras que la de los extranjeros se ubican en el centro y en los barrios de la margen derecha.

Los ciclos - circuitos- son configuraciones espaciales y funcionales constituidas por secciones con fuertes intercambios de movilidad residencial. Si bien desde el punto de vista estadístico la presencia de ciclos en ambas redes tiene una proporción similar, la localización y agrupación espacial de los ciclos difieren en ambos grupos. En el caso del grupo de españoles los ciclos más importantes forman agrupaciones complejas situadas en las periferias, distante (barrios de Santa Isabel, Montañana, San Juan de Mozarrifar, Miralbueno y urbanizaciones del noreste) y cercana (barrios Oliver y Valdefierro) y en los barrios de la margen derecha (Torreo-La Paz-Zaragoza La Vieja). En el caso de los extranjeros, los ciclos más importantes se localizan en el centro (barrio de San Pablo) y los barrios de la margen derecha Delicias y Oliver (Figura 4).

Figura 4. Centralidad-hub y ciclos de la movilidad residencial de los españoles y extranjeros, 2003-2017

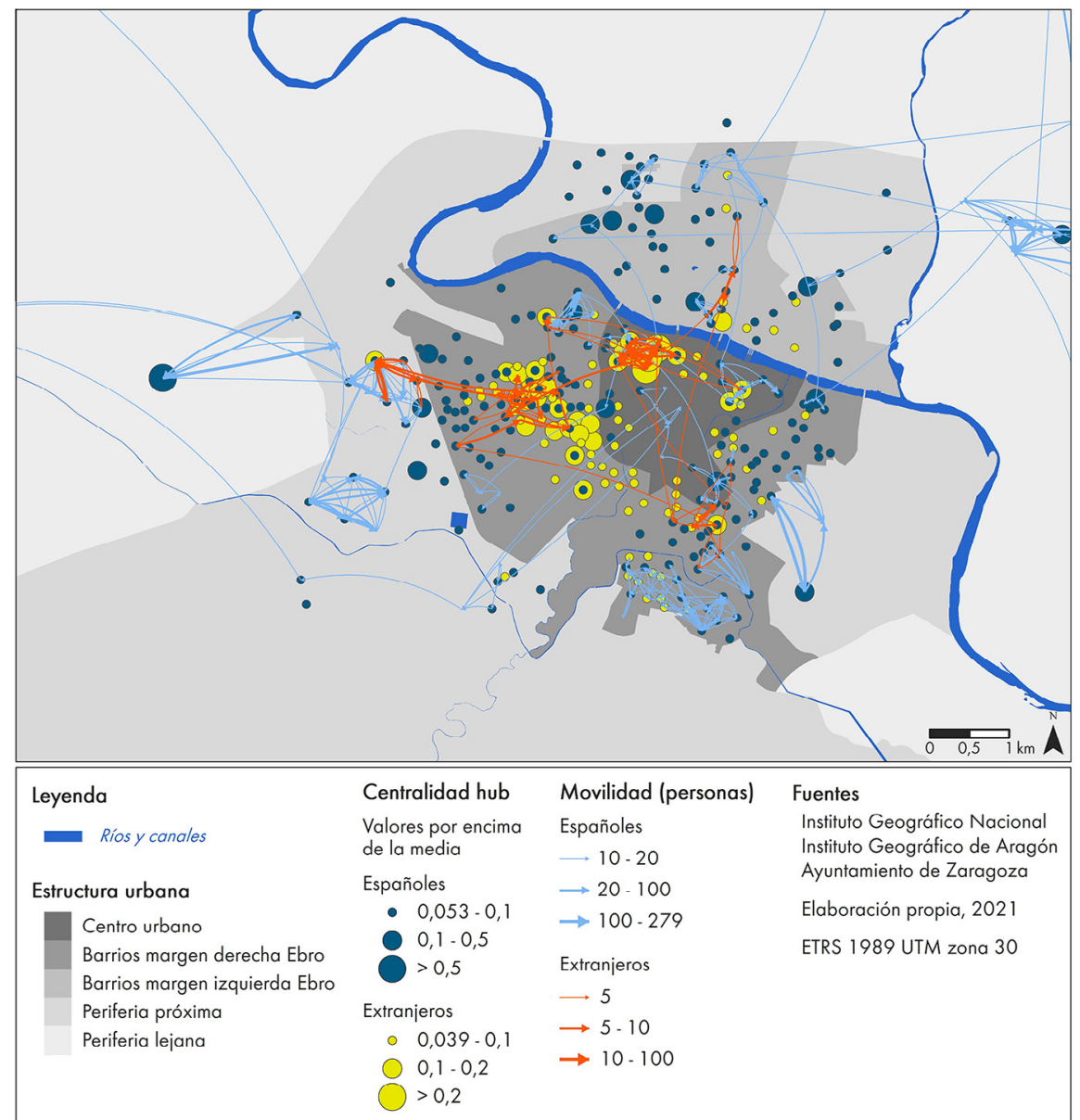

Fuente: Padrones Municipales de Habitantes del Ayuntamiento de Zaragoza. Elaboración propia 
Como se ha indicado en la metodología, los tipos de movilidad residencial se han construido a partir de los cambios de residencia entre cinco grandes áreas socioespaciales en que se ha divido la ciudad. La propensión de los residentes de cada área para cambiar de domicilio a otras áreas varía, tanto en caso de los extranjeros como en el de los españoles. Así, se observa que, en el grupo de españoles, más de la mitad de los cambios de domicilio externos con origen en los barrios de la margen derecha se dirigen a la periferia próxima; también se producen fuertes intercambios mutuos de estos barrios con el centro (Tabla 3). En el grupo de extranjeros destacan la importancia de los barrios de la margen derecha y del centro, por la movilidad interna en cada área y por los fuertes intercambios entre ambas. A la periferia próxima también llegan residentes, en particular a los barrios Oliver y Valdefierro, que proceden del barrio vecino de Delicias (margen derecha) (Tabla 2).

Tabla 2. Movilidad residencial entre áreas socioespaciales, 2003-2017

\begin{tabular}{|c|c|c|c|c|c|c|c|}
\hline \multicolumn{2}{|c|}{$\mathrm{De}->\mathrm{a}$} & \multirow{2}{*}{$\begin{array}{r}\begin{array}{l}\text { 1. Barrios } \\
\text { margen } \\
\text { derecha }\end{array} \\
29.975\end{array}$} & \multirow{2}{*}{$\begin{array}{r}\begin{array}{c}\text { 2. Barrios } \\
\text { margen } \\
\text { izquierda }\end{array} \\
5.787\end{array}$} & \multirow{2}{*}{$\begin{array}{r}\text { 3. Centro } \\
6.710\end{array}$} & \multirow{2}{*}{$\begin{array}{l}\begin{array}{l}\text { 4. Periferia } \\
\text { próxima }\end{array} \\
20.189\end{array}$} & \multirow{2}{*}{$\begin{array}{l}\text { 5. Periferia } \\
\text { lejana }\end{array}$} & \multirow{2}{*}{$\begin{array}{r}\text { Total (salen) } \\
37.982\end{array}$} \\
\hline \multirow{6}{*}{ Españoles } & 1 & & & & & & \\
\hline & 2 & 2.858 & 6.566 & 1.544 & 4.864 & 3.112 & 12.378 \\
\hline & 3 & 5.908 & 2.005 & 6.851 & 4.089 & 1.430 & 13.432 \\
\hline & 4 & 3.999 & 1.880 & 1.470 & 7.387 & 1.742 & 9.091 \\
\hline & 5 & 1.334 & 786 & 826 & 1.720 & 2.561 & 4.666 \\
\hline & Total (entran) & 14.099 & 10.458 & 10.550 & 30.862 & 11.580 & 77.549 \\
\hline \multirow{6}{*}{ Extranjeros } & 1 & 8.195 & 849 & 1.195 & 1.281 & 425 & 3.750 \\
\hline & 2 & 689 & 631 & 202 & 204 & 99 & 1.194 \\
\hline & 3 & 1.960 & 402 & 975 & 402 & 148 & 2.912 \\
\hline & 4 & 625 & 163 & 113 & 377 & 55 & 956 \\
\hline & 5 & 204 & 77 & 73 & 75 & 168 & 429 \\
\hline & Total (entran) & 3.478 & 1.491 & 1.583 & 1.962 & 727 & 9.241 \\
\hline
\end{tabular}

Fuente: Padrones Municipales de Habitantes del Ayuntamiento de Zaragoza. Elaboración propia

Los tipos de movilidad espacial también presentan diferencias entre el grupo de españoles y el de extranjeros. En ambos grupos los dos tipos de movilidad principales coinciden, pero el orden es diferente: en el caso de los españoles predominan los movimientos de suburbanización seguidos de los movientes internos en los barrios de la margen derecha, mientras que en el de los extranjeros destacan los cambios de domicilio en los barrios de la margen derecha; la segunda opción, a gran distancia, corresponde a los movimientos de suburbanización. Los movimientos de centralización tienen el mismo peso en los españoles y extranjeros, pero se trata de flujos poco numerosos en ambos grupos (no suelen superar las 10 ó 5 personas para el grupo de españoles y extranjeros respectivamente) (Tabla 3 y Figura 5). En el grupo de españoles ha alcanzado cierta importancia los cambios residenciales en el espacio periurbano, lo que indica la consolidación de un activo mercado de viviendas nuevas en la periferia de la ciudad.

Tabla 3. Tipos espaciales de movilidad residencial de los españoles y extranjeros, 2003-2017

\begin{tabular}{|c|c|c|c|c|}
\hline \multirow{2}{*}{ Movimientos } & \multicolumn{2}{|c|}{ Españoles } & \multicolumn{2}{|c|}{ Extranjeros } \\
\hline & Absolutos & Proporción (\%) & Absolutos & Proporción (\%) \\
\hline Centralización & 10.550 & 8,1 & 1.583 & 8,1 \\
\hline Internos del área-central & 6.851 & 5,2 & 975 & 5,0 \\
\hline Internos margen derecha & 29.975 & 22,9 & 8.195 & 41,8 \\
\hline Internos margen izquierda & 6.566 & 5,0 & 631 & 3,2 \\
\hline Entre barrios & 8.645 & 6,6 & 1.538 & 7,9 \\
\hline Suburbanización & 42.622 & 32,6 & 2.689 & 13,7 \\
\hline Entre periferias e internos de las periferias & 13.410 & 10,2 & 675 & 3,4 \\
\hline Total & 118.619 & 90,6 & 16.286 & 83,1 \\
\hline
\end{tabular}

Nota: el total de los valores en porcentaje no suma 100 ya que algunos tipos de movimiento no se han incluido (movimientos de las periferias a los barrios; del centro a los barrios)

Fuente: Padrones Municipales de Habitantes del Ayuntamiento de Zaragoza. Elaboración propia 
Figura 5. Movimientos residenciales de suburbanización, de centralización y movimientos internos en el centro urbano de los españoles y extranjeros, 2003-2017

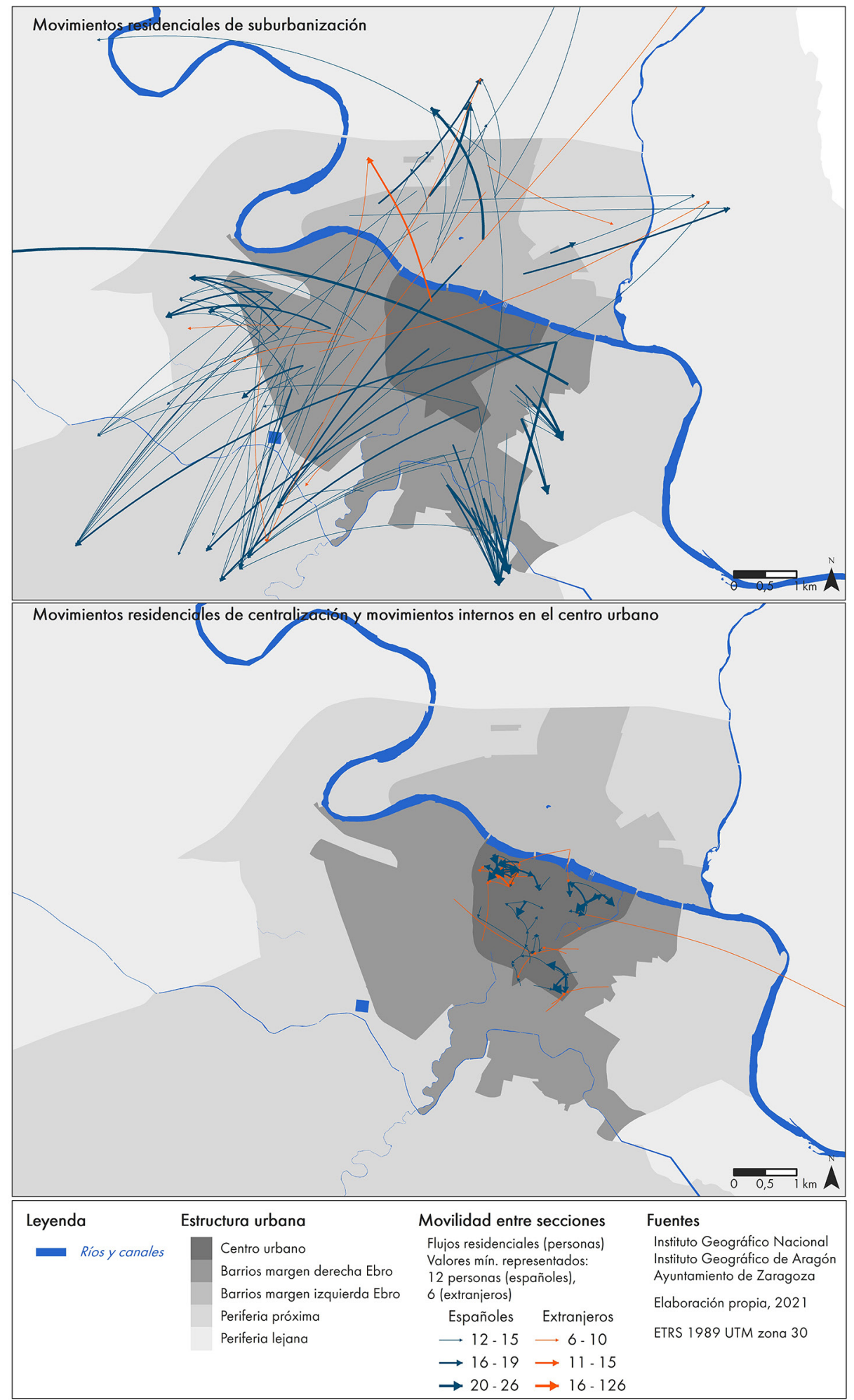

Fuente: Padrones Municipales de Habitantes del Ayuntamiento de Zaragoza. Elaboración propia

La distribución de las distancias de los cambios residenciales se aparta de la distribución nula (H0, formada por todas las distancias posibles entre las secciones), tanto en el grupo de españoles como en el de extranjeros (D de Kolmogorov-Smirnoff significativo al 0,05). 
La mayor parte de los cambios de residencia se produce a distancias muy cortas del domicilio anterior: en ambos grupos, la frecuencia máxima corresponde al tramo de 250-500 m; hasta $1 \mathrm{~km}$ de distancia se produjo casi el 30\% de la movilidad residencial de los españoles y algo más del 36\% de la de los extranjeros; hasta $3 \mathrm{~km}$ se comprende el $63 \%$ de todos los cambios residenciales de los españoles y el $77 \%$ de los extranjeros. La proporción de cambios de residencia de los españoles a menos de $1 \mathrm{~km}$ está por encima de la proporción de la distribución nula, mientras que los traslados a mayor distancia están por debajo; en el caso de los extranjeros esta distancia crítica es de 1,5 km. Las distribuciones de los diferentes tipos espaciales de movimiento en relación con sus respectivas distribuciones nulas (HO) son similares a esta tendencia general, excepto en los movimientos de suburbanización que presentan una estructura diferente, pues solamente algo más del 7\% de los movimientos residenciales de este tipo están por debajo de los $3 \mathrm{~km}$. Estos resultados se alinean con los obtenidos en otros estudios (Freeman \& Sunshine, 1976; Potrykowska, 1993; Trépanier, 1978; Bayona i Carrasco \& Pujadas Rúbies, 2010). No obstante, las distancias pueden variar de acuerdo con la resolución de las unidades espaciales utilizadas y con el tamaño físico de la ciudad.

En conjunto, las distribuciones de los cambios residenciales de los españoles y extranjeros según la distancia se ajustan muy bien a una función de distribución de densidad de probabilidad lognormal ( $\mathrm{Fi}$ gura 6), lo que permite estimar, con gran fiabilidad, la probabilidad del flujo de movilidad residencial a diferentes distancias. Así, con una probabilidad del 95\%, cabe esperar que la cuarta parte de los cambios de residencia de los españoles sea a menos de $1 \mathrm{~km}$ del domicilio actual, proporción que para esta distancia asciende al 32\% en el caso de los extranjeros; hasta $3 \mathrm{~km}$ del domicilio actual se producirían dos tercios de los cambios de residencia de los españoles $(66,5 \%)$ valor que asciende a las tres cuartas partes $(76,0 \%)$ en el caso de los extranjeros.

Figura 6. Distribución de los flujos de movilidad residencial de los españoles (izquierda) y extranjeros (derecha) según su longitud y ajuste lognormal, 2003-2017

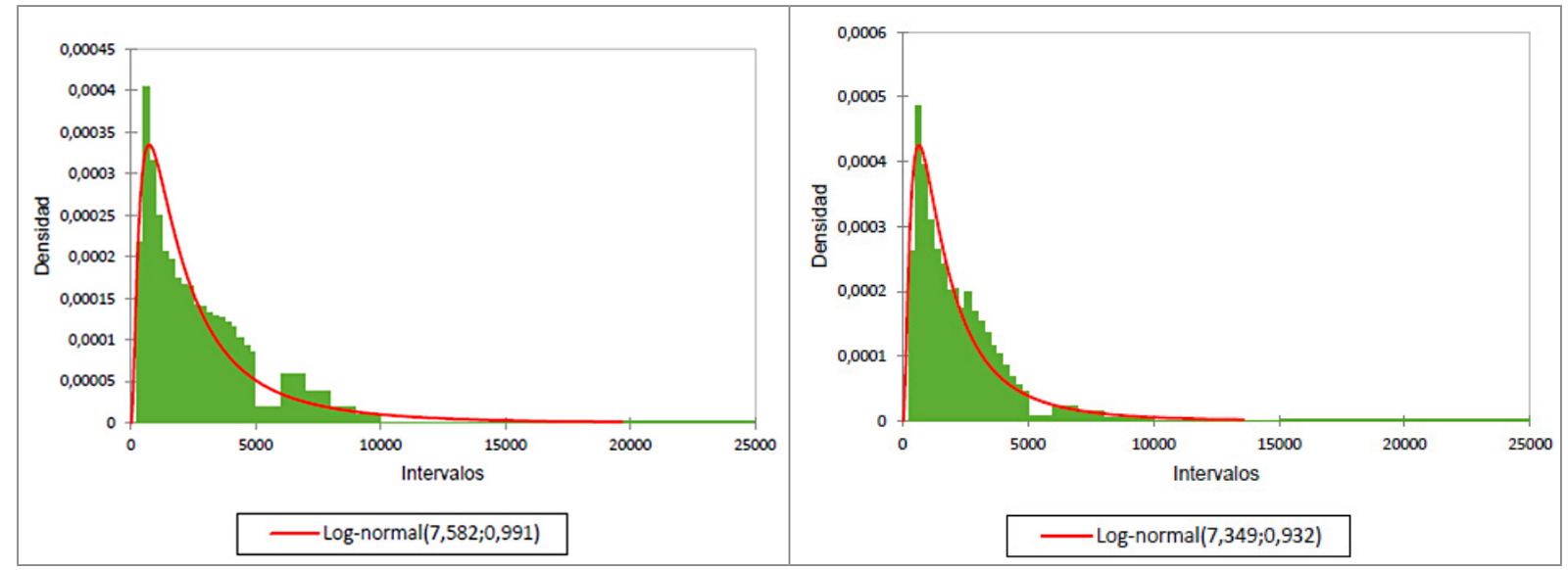

Fuente: Padrones Municipales de Habitantes del Ayuntamiento de Zaragoza. Elaboración propia

\subsection{La organización espacial de los territorios de la movilidad residencial de los españoles y extranjeros}

La división analítica de la red en módulos o grupos funcionales revela la organización de la red de flujos de la movilidad residencial y la similitud entre cada unidad (sección censal). Ambas redes poseen una estructura jerarquizada, compuesta por módulos o agrupamientos con notable cohesión interna debido a que los intercambios mutuos de residentes entre secciones del mismo módulo son bastante fuertes. La red de movilidad residencial de los españoles tiene mayor complejidad y un nivel jerárquico más que la de los extranjeros, ya que también los flujos residenciales de aquellos son mayores que los de estos.

La organización funcional se materializa en una configuración espacial diferente para ambos grupos estudiados (Tabla 4 y Figura 7). En el caso de los españoles, los agrupamientos del nivel 1 diferencian dos barrios rurales (Casetas y San Juan de Mozarrifar) del resto de la ciudad. Los clústeres de nivel 2 distinguen tres grandes áreas: dos al sur del río Ebro (margen derecha) y otra al norte de este río (margen izquierda). 
Las dos primeras compartimentan el espacio urbano en dos sectores que parten del centro, uno al este y otro hacia sur y oeste. La tercera ocupa el espacio urbano al norte del Ebro, excepto en las secciones no clasificadas, que son más abundantes en esta zona. El módulo más grande (el sector sureste, 312 secciones) muestra gran cohesión interna (índice E-I: -0,58) y fuertes intercambios con el clúster de la ribera norte.

Tabla 4. Algunas características de los módulos de la red de movilidad residencial de españoles y extranjeros de Zaragoza, 2003-2017

\begin{tabular}{|r|r|r|r|r|r|r|r|}
\hline \multicolumn{5}{|c|}{ Españoles } & \multicolumn{4}{c|}{ Extranjeros } \\
\hline Nivel & $\begin{array}{c}\text { Módulos } \\
\text { (clústeres) }\end{array}$ & $\begin{array}{c}\text { Tamaño } \\
\text { (min. -máx.) }\end{array}$ & $\begin{array}{c}\text { E-I } \\
\text { (min.; máx.) }\end{array}$ & Nivel & $\begin{array}{c}\text { Módulos } \\
\text { (clústeres) }\end{array}$ & $\begin{array}{c}\text { Tamaño } \\
\text { (min. -máx.) }\end{array}$ & $\begin{array}{c}\text { E-I } \\
\text { (min.; máx.) }\end{array}$ \\
\hline 0 & 1 & & & 0 & & & \\
\hline 1 & 3 & $2-473$ & $-0,04 ; 0,99$ & 1 & 2 & $3-482$ & 0,$21 ; 0,99$ \\
\hline 2 & 3 & $62-312$ & $-0,53 ; 0,27$ & 2 & 4 & $34-8$ & $-0,53 ; 0,17$ \\
\hline 3 & 12 & $3-116$ & $-0,14 ; 0,52$ & & & & \\
\hline
\end{tabular}

Fuente: Padrones Municipales de Habitantes del Ayuntamiento de Zaragoza. Elaboración propia

Figura 7. Agrupamientos funcionales y espaciales de la movilidad residencial y su estructura de los españoles y extranjeros, 2003-2017
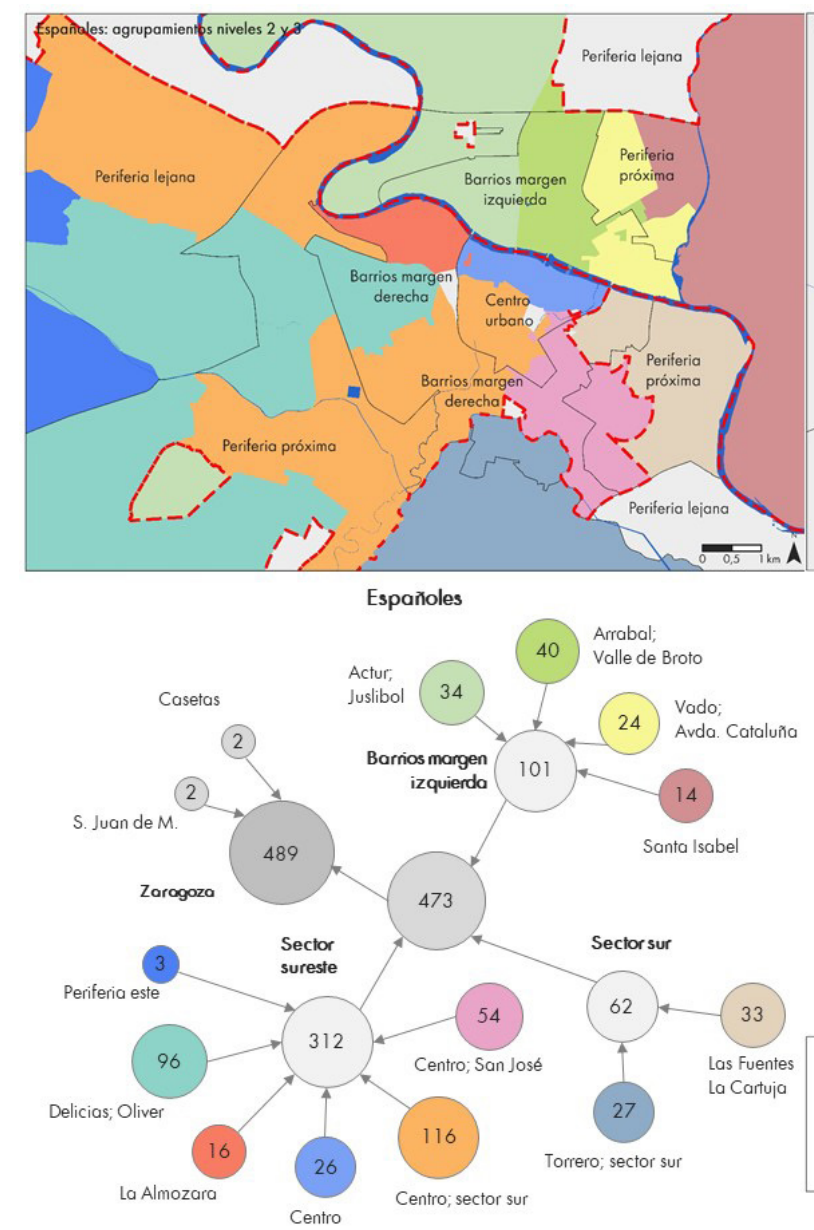

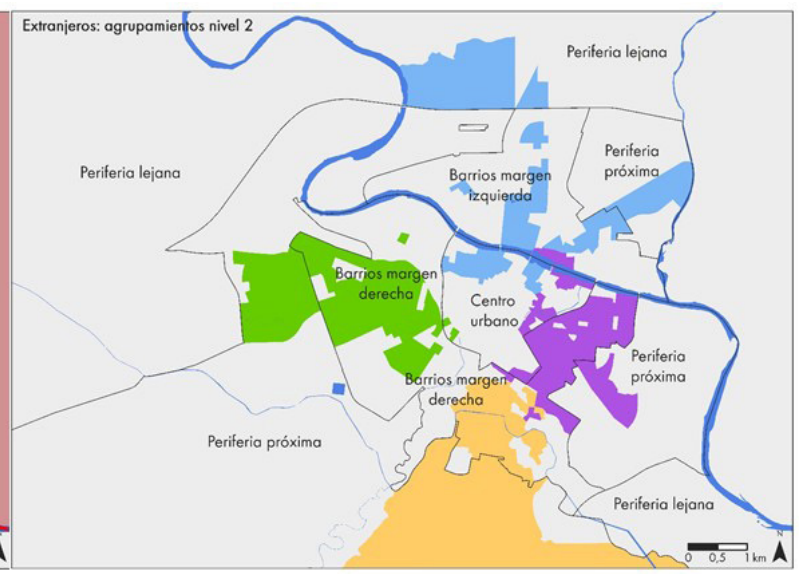

Extranjeros

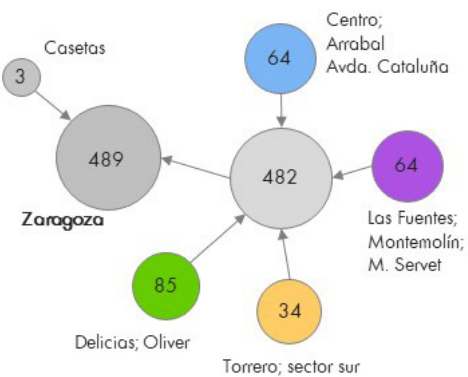

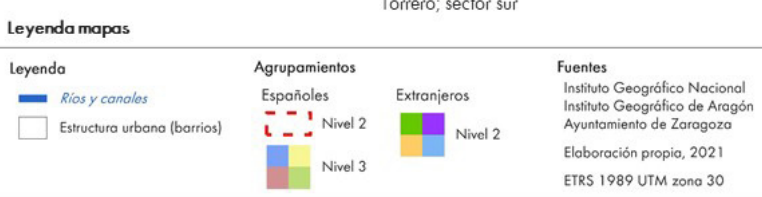

Leyenda gráficos

33) Número de secciones en cada clúster

Clúster de nivel 1 y 2 (escala de grises)

Clúster de nivel 3 (color)

Fuente: Padrones Municipales de Habitantes del Ayuntamiento de Zaragoza. Elaboración propia

En el nivel 3 se han definido doce agrupamientos de diferentes tamaños (entre 3 y 116 secciones), distintos grados de cohesión (E-I: de $-0,14$ a 0,52) y diversas configuraciones espaciales. Cabe destacar, 
en general, que todas (excepto dos) estás constituidas por secciones contiguas que tienden a formar sectores que se alargan desde el centro hacia la periferia; ciertos límites reflejan barreras físicas (el río Ebro, suelos del ferrocarril, autovías, carreteras, el río Huerva, el Canal Imperial de Aragón) que causan o refuerzan el aislamiento de algunos barrios. El efecto barrera del río Ebro se aprecia claramente en la movilidad residencial a todas las escalas. Al norte del Ebro, la carretera de Huesca (N-330) separa los barrios del ACTUR —al este- del Arrabal, Picarral, La Jota y Barrio Jesús —al oeste—; también forma un agrupamiento propio el barrio de Vadorrey; mientras que Santa Isabel y otros barrios rurales (Montañana, Movera, Peñaflor) constituyen otro clúster. Al sur del río Ebro, el barrio de La Almozara forma un clúster que refleja el relativo aislamiento de este espacio delimitado por el río Ebro y carreteras e instalaciones ferroviarias; el Casco Histórico, desde el barrio de San Pablo al de La Magdalena, conforma otro agrupamiento significativo; los barrios de Torrero-La Paz también constituyen un agrupamiento cuyos límites están reforzados por la presencia del Canal Imperial de Aragón y la ronda Z-30; el resto de los clústeres toma formas más o menos alargadas que se tienden desde el centro hacia la periferia.

La organización funcional de la red de movilidad residencial de los extranjeros se compone de dos niveles ( 1 y 2), con dos y cuatro módulos respectivamente. En el nivel 1 se diferencia el barrio rural de Casetas del resto del espacio urbano. El nivel 2 está formado por cuatro clústeres que varían también, como en el grupo anterior, en tamaño, intensidad de cohesión y configuración espacial. El clúster integrado por los barrios de Delicias y Oliver presenta una gran cohesión interna (Índice E-I: -0,53), que expresa el gran peso de los cambios residenciales entre ambos barrios y en el interior de cada uno. En general, los módulos tienen formas más irregulares y en algunos casos fragmentadas en varios espacios, que reflejan los fuertes intercambios residenciales entre secciones no adyacentes.

\section{Discusión. Movilidad residencial de los españoles y de los extranjeros: diferentes espacios, distintos procesos}

Los resultados obtenidos muestran que los cambios de residencia, tanto de los españoles como de los extranjeros, no siguen trayectorias geográficas origen-destino aleatorias si no que forman estructuras espaciales organizadas en varios niveles. Las áreas de movilidad residencial de los españoles y extranjeros se desarrollan en territorios propios, hasta cierto punto disjuntos y con características socioespaciales diferentes, pero comparten un rasgo común: en ambos casos la proximidad de las nuevas residencias al domicilio anterior constituye un elemento común del cambio de residencia. Estas características comunes y específicas sugieren que los patrones funcionales y espaciales de la movilidad de los españoles y de los extranjeros están conformados por factores y procesos también diferenciados.

La movilidad residencial de los españoles presenta dos tipos básicos de movimientos espaciales: hacia la periferia (y entre periferias) e internos en los barrios tradicionales (o entre estos barrios); ambos difieren en su rango y formas espaciales y, en las condiciones socioeconómicas de las personas que integran sus flujos. A considerable distancia de los anteriores se sitúan los movimientos de centralización e internos del área central.

Como se ha descrito, la movilidad residencial de los españoles hacia la periferia adopta configuraciones espaciales en forma de sectores continuos que se extienden desde secciones del centro hasta los límites del espacio urbano y del municipio, de tal forma que cada sector participa de espacios centrales, intermedios y periféricos. Los clústeres definidos se corresponden muy bien con los niveles de renta y tipos espaciales de movilidad. Al sur del río Ebro, el clúster central agrupa las secciones de renta más alta, que se localizan en el centro de la ciudad y hacia el sur, en los barrios Universidad, Casablanca, Montecanal, Rosales del Canal y urbanizaciones próximas al río Huerva; la movilidad residencial conecta, preferentemente, secciones del centro con las más externas, mediante flujos cuyos destinos suelen ser urbanizaciones cerradas de viviendas unifamiliares. Todos los demás clústeres, al norte y al sur del río Ebro, están constituidos por secciones de rentas medias y bajas, en los que se observan dos tipos espaciales de flujos de movilidad residencial: uno, de suburbanización y de distancia media y larga, compuesto por personas jóvenes —edad media de 31,9 años en el Distrito Sur; 35,2 años en Miralbueno; o 38,2 años en Santa Isabel frente a 43,7 años de promedio de la ciudad en 2017 (Observatorio Municipal de Estadística, 2020) - que se dirigen, en especial a nuevos ensanches residenciales con fuertes promociones de viviendas sociales (Arcosur, 21.500 viviendas; Valdespartera, 9.687 viviendas; Parque Venecia, 4.103 viviendas; Parque Goya, 3.593 viviendas); otro, de tipo interno, compuesto por un considerable flujo de personas 
que cambia el domicilio a corta distancia del actual. Esta movilidad valora las ventajas de la proximidad, ya que en el nuevo domicilio se pueden mantener la red de relaciones sociales y el uso de los equipamientos, comercios y servicios habituales, sin realizar esfuerzos suplementarios de aprendizaje y familiarización con nuevos espacios geográficos; en este dominio, la cercanía del domicilio actual y el nuevo también permite conservar el sentido de pertenencia al mismo barrio. En este grupo pueden identificarse asimismo movimientos de relocalización para grupos acomodados entre las zonas más renovadas y atractivas del Casco Histórico y de los ensanches del distrito Centro, desplazamientos que son similares a otros vistos en ciudades como Barcelona (López-Gay, 2018). Asimismo, en estos barrios se concentra la mayoría de las viviendas reconvertidas en apartamentos turísticos durante los últimos años ${ }^{5}$, en línea con un proceso análogo que afecta a los centros históricos de las ciudades españolas (De la Calle Vaquero, 2019).

En la movilidad residencial de los extranjeros predominan los cambios de domicilio a corta distancia del actual, que se producen en los espacios centrales de los barrios, especialmente de la margen derecha, en sectores del Casco Histórico y en algunos barrios rurales. Los flujos de suburbanización, a media y larga distancia, conectan áreas muy específicas de origen y destinos en la periferia: Delicias y Oliver, San José y Zaragoza La Vieja con Torrero-La Paz, sectores del centro histórico con barrios rurales, barrios rurales entre sí; estas vinculaciones sugieren, probablemente, que se trata de movimientos de reacomodación entre personas y familias de la misma nacionalidad o cultura, por razones de trabajo, reagrupación familiar y otras.

Este modelo de movilidad reúne ventajas de la cercanía del cambio de residencia con la buena accesibilidad y una estructura espacial que amplían las oportunidades de trabajo. Por una parte, la permanencia en el mismo sector permite mantener lazos sociales y familiares, así como relaciones de ayuda mutua (cuidado de niños y ancianos, hacer la compra, etc.) (Tosi, 2017). Por otra, en los centros de los barrios y en determinados sectores del Casco Histórico los alquileres son más asequibles que en otras áreas, pues se trata de espacios con la mayor parte de su caserío envejecido; además abundan locales de tamaño pequeño y mediano apropiados para negocios de autoempleo: en estas áreas son evidentes nichos étnicos para diferentes actividades (fruterías, peluquerías, bazares, locutorios, envíos de dinero, etc.) (Zhong \& Beltrán, 2020). Asimismo, la buena accesibilidad de estos espacios amplía el área en la que buscar trabajo. Estas estrategias de adaptación estimulan la movilidad residencial a corta distancia (Heylen, Mortelmans, Hermans \& Boudiny, 2012; Lauterbach, 1998; Van Diepen \& Mulder, 2009).

El patrón general hallado para la población extranjera es similar al observado en otras ciudades españolas, donde las áreas históricas degradadas y algunos barrios populares se configuran como espacios residenciales principales para este grupo (Bayona-Carrasco, 2011; Bayona-Carrasco \& López-Gay, 2011).

Una de las consecuencias inmediatas de la movilidad residencial se manifiesta en los cambios del volumen de población y de la estructura demográfica de las diferentes áreas de la ciudad. Así, buena parte de las más de 54.000 personas que fijaron su residencia en las periferias (próxima y lejana) entre 2003 y 2017, lo hicieron por cambio de su residencia de otras áreas de la ciudad (más de 32.000 cambios de residencia procedentes de otras áreas). En el mismo sentido, las pérdidas del centro urbano en este mismo período (1.327 personas) se mitigaron porque aquí se domiciliaron 6.483 personas de nacionalidad extranjera, parte de las cuales procedentes de otras áreas de la ciudad (más de 1.500 cambios residenciales entrantes en esta área). El trasiego de personas ha rejuvenecido las zonas periféricas y ha contribuido al envejecimiento de algunas áreas del centro urbano y de los barrios.

La movilidad residencial también contribuye a modificar la composición del mosaico cultural y étnico. La periferia ha diversificado el perfil de clases sociales pues, a los grupos sociales de altos ingresos residentes en urbanizaciones cerradas, se han añadido nuevos ensanches residenciales habitados por grupos de ingreso medios, e incluso bajos. En cambio, en el centro urbano y de los barrios se observa cierta homogeneización cultural y étnica debida al descenso del número de personas de nacionalidad española (Domínguez Mújica, García Coll, González Pérez \& Sánchez Aguilera, 2009) y a agrupamientos espaciales de personas de la misma nacionalidad, que según se ha observado tienden a concentrarse además en las zonas vulnerables de la ciudad ya identificadas en otros estudios (León-Casero \& Ruiz-Varona, 2017; León-Casero, 2018). Además, apenas se producen movimientos residenciales desde áreas de rentas altas al centro urbano y a los barrios tradicionales, es decir, no se observan procesos fuertes de gentrificación

5 Ebrópolis (2020), documento sin publicar. 
social, que son muy limitados todavía en Zaragoza (Cabeza Millán, 2017; Sánchez-Naudín \& Vivas-Elias, 2018; Fuertes Rodríguez, 2019).

En suma: la movilidad residencial es un vector fundamental de la suburbanización de la población de Zaragoza y de la recomposición del mosaico cultural y étnico de la ciudad, cuyos efectos son patentes en el incremento de la segregación residencial.

\section{Conclusiones}

Los resultados obtenidos se pueden generalizar del siguiente modo: a) las trayectorias origen-destino de los cambios de residencia de los españoles y de los extranjeros conforman estructuras espaciales jerarquizadas en varios niveles; b) la proximidad geográfica y la vinculación selectiva entre localizaciones disjuntas son propiedades que definen los espacios de movilidad y c) los modelos de las áreas de movilidad de los españoles y extranjeros difieren en su configuración espacial y en los factores que las generan.

Desde el punto de vista teórico, la movilidad residencial de los españoles se aproxima a un modelo de sectores (Hoyt, 1939), generado por el desplazamiento radial de las personas desde los espacios urbanos consolidados hacia la periferia, bien directamente o encadenando varios domicilios. La movilidad de los extranjeros no sigue claramente este modelo, pues los sectores, si existen, están fragmentados.

También en el ámbito de la teoría, la aportación de este trabajo corrobora una de las tendencias más constantes de la movilidad humana: el predominio de los cambios de residencia a corta distancia (Potrykowska, 1993; Ravenstein, 1885; Trépanier, 1978). Además, se han ajustado las distribuciones de las distancias de los cambios de residencia con un modelo lognormal, tanto para el grupo de españoles como para el de extranjeros. Es muy probable que este modelo de distribución se pueda generalizar a la movilidad residencial de otras muchas ciudades (e, incluso, a la movilidad en general). La forma de esta distribución es indicio de que la movilidad residencial se aproxima más a procesos de difusión que de gravedad, controlados no por la distancia física, ya que el coste económico de la mudanza de domicilio es marginal, sino por el distanciamiento social, por lo que la localización de la residencia actual es el factor que mejor predice la localización de la residencia futura.

Por otro lado, el método utilizado ha demostrado su eficacia, pues permite asociar la organización funcional y espacial de la movilidad residencial obtenidas a partir de información dinámica de los propios flujos origen-destino, en vez de utilizar datos estáticos de saldos de movilidad residencial expresados en unidades espaciales de diversos niveles.

Una vez conocidas las diferencias de la movilidad espacial entre españoles y extranjeros se propone, como línea lógica de investigación futura, extender la investigación a grupos nacionales específicos y períodos significativos, así como a grupos sociales diferenciados por la ocupación, el nivel de cualificación, el de ingresos, la edad y el tamaño de los hogares.

\section{Financiación}

Este trabajo forma parte de los resultados del proyecto de investigación "Indicadores multiescalares y herramientas cartográficas para el análisis de la vulnerabilidad socioeconómica y residencial en áreas urbanas. Aplicación al caso de Zaragoza" (CSO2016-74888-C4-3-R), del Programa Estatal de Investigación, Desarrollo e Innovación Orientada a los Retos de la Sociedad del Ministerio de Economía y Competitividad de España; en el marco del Plan Estatal de investigación Científica y Técnica y de Innovación 2017-2020.

\section{Agradecimientos}

Los autores agradecemos la provisión de datos del padrón municipal por parte del Ayuntamiento de Zaragoza en el marco de colaboración con la "Cátedra Territorio, Sociedad y Visualización Geográfica", así como el apoyo técnico prestado por Sergio Valdivielso Pardos para la preparación de la base de datos empleada en este trabajo. 


\section{Referencias}

Alonso Logroño, M. P., \& Bellet Sanfeliu, C. (2009). El tren de alta velocidad y el proyecto urbano. Un nuevo ferrocarril para la Zaragoza del tercer milenio. Scripta Nova. Revista Electrónica de Geografía y Ciencias Sociales, XIII(281). http://www.ub.edu/geocrit/sn/sn-281.htm

Andrews, D., \& Caldera Sánchez, A. (2011). Residential Mobility and Public Policy in OECD Countries. OECD Journal: Economic Studies, 2011(1), 1-22. https://doi.org/10.1787/19952856

Andris, C., Liu, X., \& Ferreira, J. (2018). Challenges for social flows. Computers, Environment and Urban Systems, 70, 197-207. https://doi.org/10.1016/j.compenvurbsys.2018.03.008

Arranz-López, A., Soria-Lara, J. A., López-Escolano, C., \& Pueyo Campos, Á. (2017). Retail Mobility Environments: A methodological framework for integrating retail activity and non-motorised accessibility in Zaragoza, Spain. Journal of Transport Geography, 58, 92-103. https://doi.org/10.1016/j. jtrangeo.2016.11.010

Bayona-Carrasco, J. (2011). La movilidad intraurbana de los extranjeros en Barcelona. Architecture, City and Environment, VI(17), 129-156. https://doi.org/10.5821/ace.v6i17.2532

Bayona i Carrasco, J., \& Pujadas Rúbies, I. (2010). Cambios residenciales internos en la ciudad de Barcelona: evolución y características territoriales. Investigaciones Geográficas, 52, 9-36. https://doi. org/10.14198/INGEO2010.52.01

Bayona-Carrasco, J., \& López-Gay, A. (2011). Concentración, segregación y movilidad residencial de los extranjeros en Barcelona. Documents d'Anàlisi Geogràfica, 57(3), 381-412. https://doi.org/10.5565/ rev/dag. 234

Bayona-Carrasco, J., \& Gil-Alonso, F. (2012). Suburbanization and international migration: the case of the Barcelona metropolitan region (1998-2009). Tijdschrift Voor Economische En Sociale Geografie, 103(3), 312-329. https://doi.org/10.1111/j.1467-9663.2011.00687.x

Bible, D. S., \& Brown, L. A. (1981). Place utility, attribute tradeoff, and choice behavior in an intra-urban migration context. Socio-Economic Planning Sciences, 15(1), 37-44. https://doi.org/10.1016/00380121(81)90016-1

Bonacich, P. (1972). Factoring and weighting approaches to status scores and clique identification. The Journal of Mathematical Sociology, 2(1), 113-120. https://doi.org/10.1080/0022250X.1972.9989806

Brown, L. A., \& Moore, E. G. (1970). The Intra-Urban Migration Process: A perspective. Geografiska Annaler. Series B, Human Geography, 52, 1-13. https://doi.org/10.1080/04353684.1970.11879340

Brummel, A. C. (1977). A theory of intraurban residential mobility behaviour. Recuperado de https:// macsphere.mcmaster.ca/handle/11375/12914\#?

Cabeza Millán, L. (2017). Procesos de gentrificación urbana. Barrio de la Magdalena. Zaragoza (Trabajo Fin de Grado). Universidad de Zaragoza. Recuperado de https://zaguan.unizar.es/record/65361/files/ TAZ-TFG-2017-1806.pdf

Callizo-Soneiro,J. (2017). La ville espagnole à l'âge post-industriel: De la crise du « fordisme »à l'éclatement de la bulle immobilière. Méditerranée, 129, 73-90. https://doi.org/10.4000/mediterranee.9222

Carley, K. M. (2017). ORA-NET SCENES. Center for Computational Analysis of Social and Organizational Systems (CASOS), Institute for Software Research International (ISRI), School of Computer Science, Carnegie Mellon University.

Clark, W. A. V., \& Dieleman, F. M. (1996). Households and housing: choice and outcomes in the housing market. New Brunswick, N.J: Center for Urban Policy Research.

Coulter, R., Ham, M. van, \& Findlay, A. M. (2015). Re-thinking residential mobility: Linking lives through time and space. Progress in Human Geography, 40(3), 352-374. https://doi. org/10.1177/0309132515575417

De la Calle Vaquero, M. (2019). Turistificación de centros urbanos: clarificando el debate. Boletín de la Asociación de Geógrafos Españoles, 83(2829), 1-40. https://doi.org/10.21138/bage.2829

De Miguel González, R. (2015). Transformación urbana y procesos territoriales recientes en Zaragoza y su espacio metropolitano. Estudios Geográficos, 76(278), 63-106. https://doi.org/10.3989/ estgeogr.201503 
Di Virgilio, M. M. (2011). La movilidad residencial: una preocupación sociológica. Territorios, 25, $173-190$. Recuperado de https://revistas.urosario.edu.co/index.php/territorios/article/view/1851

Domínguez Mújica, J., García Coll, A., González Pérez, J. M., \& Sánchez Aguilera, D. (2009). La population étrangère en Espagne: Quelques éléments d’une géographie changeante. Sud-Ouest Européen, 26, 71-88.

Ducruet, C., \& Beauguitte, L. (2014). Spatial Science and Network Science: Review and Outcomes of a Complex Relationship. Networks and Spatial Economics, 14(3-4), 297-316. https://doi.org/10.1007/ s11067-013-9222-6

Ebrópolis. (2019). Estrategia Zaragoza +20. Recuperado de http://www.ebropolis.es/files/File/Estrategia20/ ESTRATEGIA/Estrategia\%20Zaragoza+20-Ebropolis-2019.pdf

Escolano-Utrilla, S., López-Escolano, C., \& Pueyo-Campos, Á. (2018). Urbanismo neoliberal y fragmentación urbana: el caso de Zaragoza en los primeros quince años del siglo XXI. EURE-Revista Latinoamericana de Estudios Urbano Regionales, 44(132), 183-210. https://doi.org/10.4067/s0250$\underline{71612018000200185}$

Freeman, L. C., \& Sunshine, M. H. (1976). Race and Intra-Urban Migration. Demography, 13(4), 571575. https://doi.org/10.2307/2060511

Fuertes Rodríguez, V. (2019). Economías alternativas y gentrificación: el caso de la Magdalena (Zaragoza) (Trabajo Fin de Grado). Universidad de Zaragoza. Recuperado de https://zaguan.unizar.es/ record/87977/files/TAZ-TFG-2019-4304.pdf

Gómez Bahillo, C., \& Sanaú Villarroya, J. (2009). La Exposición Internacional Zaragoza 2008. CESA, Consejo Económico y Social de Aragón.

Heylen, L., Mortelmans, D., Hermans, M., \& Boudiny, K. (2012). The intermediate effect of geographic proximity on intergenerational support: A comparison of France and Bulgaria. Demographic Research, 27, 455-486. https://doi.org/10.4054/DemRes.2012.27.17

Hoyt, H. (1939). The Structure and Growth of Residential Neighborhoods in American Cities. Washintong, D.C.: Federal Housing Administration.

Jia, X., \& Lei, J. (2019). Residential Mobility of Locals and Migrants in Northwest Urban China. Sustainability, 11(13), 3507. https://doi.org/10.3390/su11133507

Jiménez, V., Hidalgo, R., Campesino, A.-J., \& Alvarado, V. (2018). Normalización del modelo neoliberal de expansión residencial más allá del límite urbano en Chile y España. EURE-Revista Latinoamericana de Estudios Urbano Regionales, 44(132), 27-46. https://doi.org/10.4067/s0250-71612018000200027

King, R. (2012). Geography and Migration Studies: Retrospect and Prospect. Population, Space and Place, 18(2), 134-153. https://doi.org/10.1002/psp.685

Kleinberg, J. M. (1998). Decision algorithms for unsplittable flow and the half-disjoint paths problem. In: Proceedings of the thirtieth annual ACM symposium on Theory of computing, ACM Press (pp. 530-539). https://doi.org/10.1145/276698.276867

Lancichinetti, A., Radicchi, F., Ramasco, J. J., \& Fortunato, S. (2011). Finding Statistically Significant Communities in Networks. PLoS ONE, 6(4), e18961. https://doi.org/10.1371/journal.pone.0018961

Lauterbach, W. (1998). Die Multilokalität später Familienphasen. Zur räumlichen Nähe und Ferne der Generationen. Zeitschrift für Soziologie, 27(2), 113-132. https://doi.org/10.1515/zfsoz-1998-0203

León-Casero, J. (2018). Mapa de Riesgo Social de Zaragoza: herramientas complementarias para medir el potencial regenerativo de zonas vulnerables. Ciudad y Territorio. Estudios Territoriales, L(197), 503-516. Recuperado de https://recyt.fecyt.es/index.php/CyTET/article/view/76679

León-Casero, J., \& Ruiz-Varona, A (2017). Estrategias de desarrollo urbano sostenible: inclusión social y regeneración urbana en consenso obligado. Zaragoza como caso de estudio. Ciudades, 20, 111-134. https://doi.org/10.24197/ciudades.20.2017.24

Liu, X., Hollister, R., \& Andris, C. (2018). Wealthy Hubs and Poor Chains: Constellations in the U.S. Urban Migration System. En: L. Perez, E.-K. Kim, \& R. Sengupta (Eds.), Agent-Based Models and Complexity Science in the Age of Geospatial Big Data (pp. 73-86). Cham: Springer International Publishing. Recuperado de http://link.springer.com/10.1007/978-3-319-65993-0_6

López-Gay, A. (2014). Population growth and re-urbanization in Spanish inner cities: The role of internal migration and residential mobility. Revue Quetelet/Quetelet Journal, 2(1), 67-92. https://doi. org/10.14428/rqj2014.02.01.03 
López-Gay, A. (2017). Hacia un patrón territorial complejo de la movilidad residencial. El caso de la Región Metropolitana de Barcelona. Papers. Revista de Sociologia, 102(4), 793-823. https://doi. org/10.5565/rev/papers.2420

López-Gay, A. (2018). Cambio en la composición social y gentrificación en Barcelona: una mirada a través de los flujos migratorios y residenciales. Papers. Revista de Sociologia, 60, 80-93. https://iermb.uab. cat/ca/revistapapers/n-60-gentrificacio-i-dret-a-la-ciutat/\#close

Luo, W., \& MacEachren, A. M. (2014). Geo-social visual analytics. Journal of Spatial Information Science, 8, 27-66. https://doi.org/10.5311/JOSIS.2014.8.139

Malliaros, F. D., \& Vazirgiannis, M. (2013). Clustering and community detection in directed networks: A survey. Physics Reports, 533(4), 95-142. https://doi.org/10.1016/j.physrep.2013.08.002

Martori, J. C., Madariaga, R., \& Oller, R. (2016). Real estate bubble and urban population density: six Spanish metropolitan areas 2001-2011. The Annals of Regional Science, 56(2), 369-392. https://doi. org/10.1007/s00168-016-0743-z

Méndez, M. L., \& Gayo, M. (2019). Social Mobility over Time and in Space: Ascending Residential and Social Trajectories. En: Upper Middle Class Social Reproduction (pp. 29-54). Cham: Springer International Publishing. Recuperado de http://link.springer.com/10.1007/978-3-319-89695-3_2

Morris, T., Manley, D., \& Sabel, C. E. (2018). Residential mobility: Towards progress in mobility health research. Progress in Human Geography, 42(1), 112-133. https://doi.org/10.1177/0309132516649454

Murayama, Y., Inoue, T., \& Hashimoto, Y. (1997). Spatial chain patterns of intra-urban migration. Geographia Polonica, 69, 135-152.

Observatorio Municipal de Estadística (2020). Datos demográficos de Zaragoza. Recuperado de http:// demografia.zaragoza.es

Potrykowska, A. (1993). Intra-urban migration in the Warsaw urban region. Geographia Polonica, 61, 281-292.

Pujadas Rúbies, I., Prats Boned, P., \& Coll Noguera, M. (2007). Elección residencial y nuevas formas urbanas: el caso de la Región Metropolitana de Barcelona. En A. A. Artigues, A. Bauzà, M. Blázquez, J. M. González, I. Murray, \& O. Rullán (Eds.), Los procesos urbanos postfordistas (pp. 215-234). Palma de Mallorca: Universitat de les Illes Balears y AGE.

Quigley, J. M., \& Weinberg, D. H. (1977). Intra- Urban Residential Mobility: A Review and Synthesis. International Regional Science Review, 2(1), 41-66. https://doi.org/10.1177/016001767700200104

Ratti, C., Sobolevsky, S., Calabrese, F., Andris, C., Reades, J., Martino, M., ... \& Strogatz, S. H. (2010). Redrawing the Map of Great Britain from a Network of Human Interactions. PLoS ONE, 5(12), e14248. https://doi.org/10.1371/journal.pone.0014248

Raulin, A. (2014). Anthropologie urbaine. Paris: Armand Colin.

Ravenstein, E. G. (1885). The Laws of Migration. Journal of the Royal Statistical society, 48, 167-227. https://doi.org/10.2307/2979181

Sánchez-Naudín, J., \& Vivas-Elias, P. (2018). La ciudad creativa y cultural como espacio de exclusión y segregación. Analizando La Placica Vintage de Zaragoza: materialidades, prácticas, narrativas y virtualidades. EURE-Revista Latinoamericana de Estudios Urbano Regionales, 44(133), 211-232. https:// doi.org/10.4067/s0250-71612018000300211

Shannon, P., Markiel, A., Baliga, N., Wang, J., Ramage, D., Amin, N., .. \& Ideker, T. (2003). Cytoscape: a software environment for integrated models of biomelecular interaction networks. Genome Research, 13(11), 2498-504. https://doi.org/10.1101/gr.1239303

Sun, S., \& Manson, S. M. (2012). Intraurban Migration, Neighborhoods, and City Structure. Urban Geography, 33(7), 1008-1029. https://doi.org/10.2747/0272-3638.33.7.1008

Szalay-Beko, M., Palotai, R., Szappanos, B., Kovacs, I. A., Papp, B., \& Csermely, P. (2012). ModuLand plug-in for Cytoscape: Determination of hierarchical layers of overlapping network modules and community centrality. Bioinformatics, 28(16), 2202-2204. https://doi.org/10.1093/bioinformatics/ $\underline{\text { bts352 }}$

Thiers-Quintana, J., \& Gil-Alonso, F. (2019). Dinámicas residenciales de la inmigración latinoamericana en las metrópolis de Barcelona y Madrid: cambios de tendencias durante la expansión, la crisis y la poscrisis. Documents d'Anàlisi Geogràfica, 66(1), 57-82. https://doi.org/10.5565/rev/dag.584 
Torrado, J. M. (2020). Flujos espaciales y dinámicas residenciales de centralización en las áreas metropolitanas españolas (Tesis doctoral). Universidad de Granada. Recuperado de http://hdl.handle.net/10481/59770

Tosi, M. (2017). Leaving-home transition and later parent-child relationships: proximity and contact in Italy. European Societies, 19(1), 69-90. https://doi.org/10.1080/14616696.2016.1226374

Trépanier, C. (1978). La mobilité dans l'agglomération de Trois-Rivières. Cahiers de géographie du Québec, 22(57), p. 377-392. https://doi.org/10.7202/021410ar

Van der Vlist, A. J., Czamanski, D., \& Folmer, H. (2011). Immigration and urban housing market dynamics: the case of Haifa. The Annals of Regional Science, 47(3), 585-598. https://doi.org/10.1007/ s00168-010-0396-2

Van Diepen, A. M. L., \& Mulder, C. H. (2009). Distance to family members and relocations of older adults. Journal of Housing and the Built Environment, 24(1), 31-46. https://doi.org/10.1007/s10901-008-9130-0

Villani, T. (2014). Psychogéographies urbaines corps, territoires et technologies. Paris: Eterotopia France.

White, P. (1985). Levels of intra-urban migration in western european cities. Espace, populations, sociétés, 3(1), 161-169. https://doi.org/10.3406/espos.1985.1024

Zhong, W., \& Beltrán, J. (2020). Vivienda y movilidad. comportaminto residencial de la migración china en España. Scripta Nova. Revista Electrónica de Geografía y Ciencias Sociales, XXV(629), p. 28. https:// doi.org/10.1344/sn2020.24.22730 